\title{
Treat-and-extend therapy using intravitreal aflibercept for neovascular age-related macular degeneration: 2-year real-world practice data from Slovenia
}

\author{
Polona Jaki Mekjavić ${ }^{1,2^{*}}$ (D), Bogdan Gregorčič ${ }^{3}$, Cvetka Oberč ${ }^{4}$ and Slava Podgoršek ${ }^{5}$
}

\begin{abstract}
Background: To assess visual outcomes over 24 months in patients with neovascular age-related macular degeneration (nAMD) who initiated intravitreal aflibercept therapy under a treat-and-extend (TE) regimen in real-world settings.

Methods: In this retrospective, observational, multicentre study in Slovenia, medical records of all treatmentnaïve patients with nAMD who started intravitreal aflibercept therapy between October 2013 and April 2015 were reviewed. The primary outcome measure was change in mean visual acuity (VA) from baseline to 24 months in patients who received the TE regimen for 2 years, assessed by standardised Early Treatment Diabetic Retinopathy Study charts and calculated as least-squares means. Other outcome measures included the numbers of injections and visits at 12 months and 24 months.

Results: The primary analysis included 115 eyes of 105 patients who received TE treatment for 2 years (Group A). The mean VA improved from $57.9 \pm 14.9$ letters at baseline to $64.6 \pm 15.8$ letters $(+6.5$ letters, $p<0.0001)$ at 12 months and $64.8 \pm 15.6$ letters $(+7.0$ letters, $p<0.0001)$ at 24 months. The mean number of injections per eye was $8.4 \pm 1.9$ and the mean number of visits was $8.8 \pm 1.7$ at 12 months; these numbers decreased to 6.1 \pm 2.0 and $6.4 \pm 1.9$, respectively, at 24 months. The additional analysis included 33 eyes of 33 patients who received TE treatment in Year 1, followed by pro re nata treatment in Year 2 (Group B). Compared with Group A whose vision improvement was maintained at 24 months, the VA gain in Group B eyes seen at 12 months (change in mean VA vs baseline: +6.9 letters, $p=0.0008$ ) was no longer present at 24 months (change in mean VA vs baseline: +1.2 letters, $p=0.5733$ ).

Conclusions: Using the TE regimen in clinical practice, intravitreal aflibercept significantly improved visual outcomes in treatment-naïve patients with $\mathrm{nAMD}$, which were maintained over time. TE therapy with intravitreal aflibercept is a rational long-term strategy that can produce favourable outcomes in clinical practice.
\end{abstract}

Keywords: Aflibercept, Anti-VEGF, Neovascular age-related macular degeneration, Real-world data, Treat and extend, Visual acuity

\footnotetext{
* Correspondence: polona.jaki@guest.arnes.si

${ }^{1}$ Eye Hospital, University Medical Center Ljubljana, Grabloviceva 46, SI-1000

Ljubljana, Slovenia

${ }^{2}$ Medical Faculty, University of Ljubljana, Ljubljana, Slovenia

Full list of author information is available at the end of the article
}

(c) The Author(s). 2018 Open Access This article is distributed under the terms of the Creative Commons Attribution 4.0 International License (http://creativecommons.org/licenses/by/4.0/), which permits unrestricted use, distribution, and reproduction in any medium, provided you give appropriate credit to the original author(s) and the source, provide a link to the Creative Commons license, and indicate if changes were made. The Creative Commons Public Domain Dedication waiver (http://creativecommons.org/publicdomain/zero/1.0/) applies to the data made available in this article, unless otherwise stated. 


\section{Background}

Age-related macular degeneration (AMD) is one of the leading causes of visual impairment in industrialised countries [1]. The advanced stage of the disease, neovascular AMD (nAMD), is responsible for the most severe vision loss and can have a debilitating effect on quality of life [2, 3]. The unravelling of the core molecular mechanisms of nAMD has led to the approval of two robust anti-vascular endothelial growth factor (VEGF) therapies which have now become the standard of care $[4,5]$. Findings from the pivotal ranibizumab studies ANCHOR and MARINA [6, 7], and aflibercept studies VIEW 1 and VIEW $2[8,9]$, have shown clinical benefits of these anti-VEGF agents in improving visual acuity (VA), preventing loss of vision or maintaining vision in most patients with nAMD.

In addition to the fixed-dosing protocol during the first year as per label [4], pro re nata (PRN) and treat-and-extend (TE) are also used for intravitreal aflibercept injections in clinical practice [10]. Initially described by Spaide in 2007 [11], the individually tailored TE approach is gaining popularity among retinal specialists to minimise the need for frequent treatment and the burden on patients [12]. Due to its earlier availability, ranibizumab treatment using a TE strategy for nAMD has been investigated in both randomised controlled trials (RCTs) and real-world studies, and has been reported to be comparable with fixed-dosing regimens and either equivalent or superior to PRN protocols [13-17]. However, limited information is available on TE approaches in nAMD using intravitreal aflibercept: a small prospective trial and a retrospective database study provide evidence that intravitreal aflibercept TE therapy can produce good outcomes over 2 years while reducing the treatment burden $[18,19]$.

Intravitreal aflibercept was first registered in Slovenia in November 2012 [4] and was used for the treatment of nAMD in four centres in the country at the time of this study. This retrospective analysis reported the 24-month VA outcomes in treatment-naïve patients with nAMD using intravitreal aflibercept in routine clinical practice from all four centres in Slovenia. The primary objective of the study was to assess visual outcomes over 24 months in patients who received intravitreal aflibercept therapy under a TE regimen.

\section{Methods}

A retrospective, observational, multicentre study of nAMD patients in Slovenia over a 2 -year period was conducted after requesting the approval of the National Medical Ethics Committee of Slovenia. We retrospectively identified in our medical records all patients who started treatment with intravitreal aflibercept between October 2013 and April 2015 for the indication of treatment-naïve nAMD in at least one eye. Patient documentation was reviewed and data collected at 4, 12, 18 and 24 months. In total, four sites in Slovenia participated in this study: Eye Hospital University Medical Center Ljubljana, General Hospital "Dr Franca Derganca" Nova Gorica, General Hospital Nova Mesto, and General Hospital Celje. All patients included in this study provided written informed consent to the use of their anonymised data for the purposes of clinical audit and research, as per participating hospital policy.

We reviewed a series of consecutive cases of 166 patients (182 eyes) with active nAMD who were offered treatment with intravitreal aflibercept. Diagnosis was confirmed by clinical examination (slit-lamp fundoscopy), spectral domain optical coherence tomography (SD-OCT) and angiography. Inclusion criteria included treatment-naïve active nAMD, proven/diagnosed with fluorescein and/or indocyanine green angiography. Different types of active lesions of all sizes were included: classic, occult, minimally classic and retinal angiomatous proliferation; polypoid lesions were excluded.

Patients received intravitreal aflibercept following one of the three protocols: 1) TE for 2 years; 2) TE for 1 year and then switching to PRN the following year; 3) PRN for 2 years. All patients received consecutive injections of intravitreal aflibercept at 4- to 5 -week intervals until an anatomical improvement of the macula was apparent, as noted by SD-OCT as a reduction in intraretinal and subretinal fluid. When the macula became dry, in patients following the TE protocol, there was a stepwise prolongation of treatment intervals of 2 weeks up to 14 weeks. If a recurrence of subretinal and/or intraretinal fluid or new haemorrhage was evident, the treatment interval was reduced by 2 weeks. Patients were examined and treated on the same day. Patients following the PRN protocol had bimonthly follow-up and received additional reinjections if any of the following changes were observed by the evaluating physician as shown: (1) VA loss of at least 5 letters with SD-OCT evidence of subretinal and/or intraretinal fluid, (2) new macular haemorrhage, or (3) evidence of persistent/ increased subretinal and/or intraretinal fluid on $\mathrm{SD}-\mathrm{OCT}$ at least 1 month after the previous injection. All retreatment criteria were based by comparing the results with the previous visit.

A shared decision-making approach based on both the patients' and the treating ophthalmologists' preferences was used to determine the treatment protocol before treatment initiation and during the annual review. The TE protocol was used if receiving treatment on each visit with possible extension of treatment intervals was preferred, whereas PRN was used if more frequent monitoring and less injections were preferred. 
On each visit, patients were questioned about any adverse events (AEs) observed between visits. VA was measured by standardised Early Treatment Diabetic Retinopathy Study (ETDRS) charts; SD-OCT (Topcon or Heidelberg) and slit-lamp fundoscopy were performed.

The primary analysis included eyes that received TE treatment for 2 years (Group A) and the primary measure was change in mean VA from baseline to 24 months. Other measurements included the mean number of injections per eye, the mean number of visits and the proportions of eyes stratified by VA at 4, 12 and 24 months. Additional analysis included comparison of VA outcomes between Group A eyes and those switched from the TE to PRN regimen in Year 2 (Group B).

\section{Statistical analysis}

Calculations were performed with SAS Version 9.4 (SAS Institute Inc., Cary, NC, USA).

If not otherwise stated, all values are presented as mean \pm standard deviation. VA measurements were analysed using mixed models with repeated measures to account for the availability of two measurements (both eyes) for some patients. The VA at a given time point was modelled as a dependent variable, while baseline VA was used as an independent variable. An unstructured covariance structure was chosen and the patients constituted a repeated factor. To investigate the two different treatment regimens, the regimen was used as an additional fixed factor in an extended model. Least-squares means and their 95\% confidence intervals were utilised to estimate mean absolute values, mean absolute changes and mean differences in changes. $P$-values of the corresponding type 3 tests of fixed effects were considered as statistically significant when they were below 0.05 .

\section{Results}

\section{Baseline characteristics}

The final analysis included 115 eyes of 105 patients who received TE treatment for 2 years (Group A) and 33 eyes of 33 patients who received TE treatment for 1 year and PRN treatment for the following year (Group B), excluding 30 eyes of 24 patients treated with PRN aflibercept for 2 years and 4 eyes of 4 patients that were lost to follow-up. The baseline characteristics of all patients included in the study are summarised in Table 1. The demographic characteristics between Group A and Group B eyes were comparable.

\section{Primary analysis}

The mean VA over the course of the study in Group A eyes is illustrated in Fig. 1. Scatterplots of VA at baseline vs VA at 4, 12, 18 and 24 months are shown in Fig. 2 A$\mathrm{D}$, respectively. The mean VA and change in mean VA
Table 1 Patient demographics and baseline characteristics

\begin{tabular}{llll}
\hline & Group A & Group B & p-value \\
\hline Number of patients & 105 & 33 & \\
Number of eyes & 115 & 33 & \\
Female/Male & $67 / 38$ & $20 / 13$ & $0.8367^{\mathrm{a}}$ \\
Age (years, mean \pm SD) & $77.3 \pm 7.3$ & $76.3 \pm 8.4$ & $0.5303^{\mathrm{b}}$ \\
VA (by eye, mean \pm SD) & $57.9 \pm 14.9$ & $62.4 \pm 12.4$ & $0.0850^{\mathrm{b}}$ \\
\hline
\end{tabular}

${ }^{a}$ Fisher's exact test; ${ }^{b} \mathrm{t}$-test assuming unequal variances (Satterthwaite method) Group A: eyes receiving the TE regimen for 2 years; Group B: eyes receiving the TE regimen in Year 1 and the PRN regimen in Year 2. PRN: pro re nata; SD: standard deviation; TE: treat-and-extend; VA: visual acuity

vs baseline are detailed in Table 2. After 12 months, $48.7 \%$ of eyes had VA of $\geq 70$ letters (Table 3); the mean number of injections per eye was $8.4 \pm 1.9$ and the mean number of visits was $8.8 \pm 1.7$. After 24 months, $43.5 \%$ of eyes had VA of $\geq 70$ letters; the mean number of injections per eye and the mean number of visits decreased to $6.1 \pm 2.0$ and $6.4 \pm 1.9$, respectively.

\section{Additional analysis}

The mean VA and change in mean VA vs baseline in Group B eyes at 4, 12, 18 and 24 months are shown in Table 2. After 12 months, $57.6 \%$ of eyes had VA of $\geq 70$ letters; the mean number of injections per eye was $7.8 \pm$ 1.4 and the mean number of visits was $8.1 \pm 1$.4. After 24 months, $51.5 \%$ of eyes had VA of $\geq 70$ letters; the mean number of injections per eye and the mean number of visits decreased to $2.5 \pm 1.7$ and $6.2 \pm 1.8$, respectively.

When comparing Group B with Group A, the differences in mean VA change from baseline to 4 months and to 12 months were negligible. At 18 months, there was a small, non-statistically significant difference in mean VA change from baseline (1.7 letters, $p=0.4952$ ) favouring Group A; this difference increased to 5.7 letters with statistical significance at 24 months ( $p=$ 0.0224).

\section{Ocular safety}

The following ocular-specific AEs were observed: endophthalmitis $(n=1$, severe; treated with vitrectomy and intravitreal antibiotics); non-infectious inflammation $(n=1$, severe; treated with local corticosteroids for several days); dehiscence of conjunctiva on the injection site $(n=2$, both mild; treated with local antibiotic ointment for a few days). Vitreous opacities (bubbles) were anecdotally reported and considered mild. Precise numbers were not tabulated.

\section{Discussion}

Over the past years, researchers and clinicians have been seeking ways to minimise the frequency of treatment for nAMD patients receiving anti-VEGF therapy without compromising visual outcomes [12]. Positive clinical 


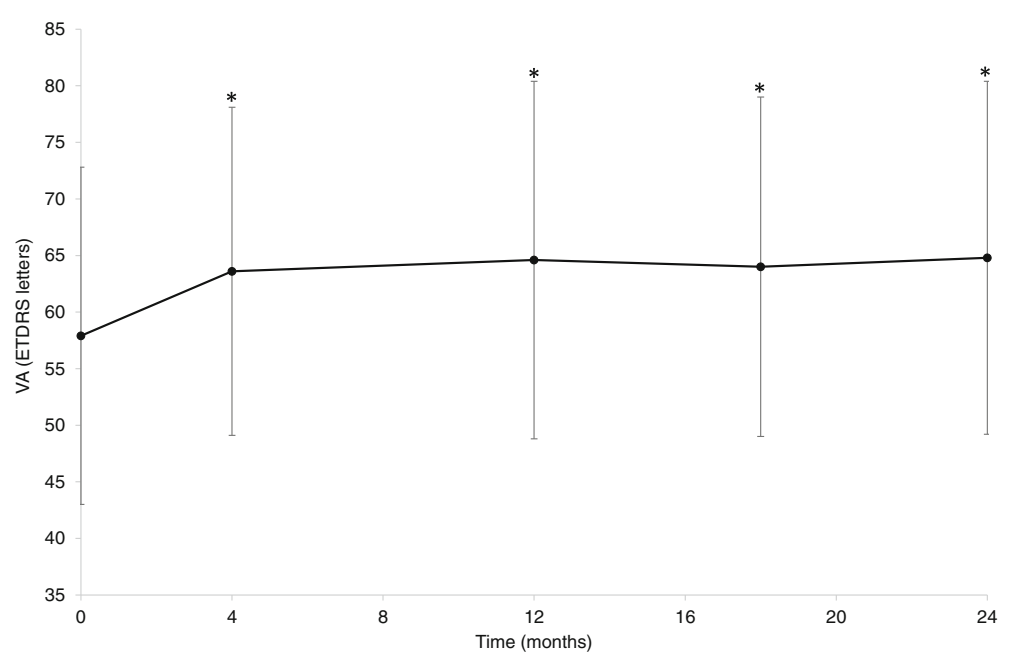

Fig. 1 Mean VA \pm SD from baseline to 24 months in eyes receiving treat-and-extend aflibercept for 2 years $(n=115){ }^{*} p<0.05$. VA values refer to least-squares means. ETDRS: Early Treatment Diabetic Retinopathy Study; SD: standard deviation; VA: visual acuity

A

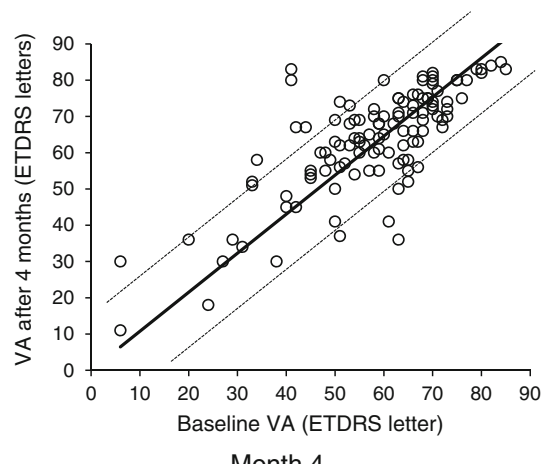

C

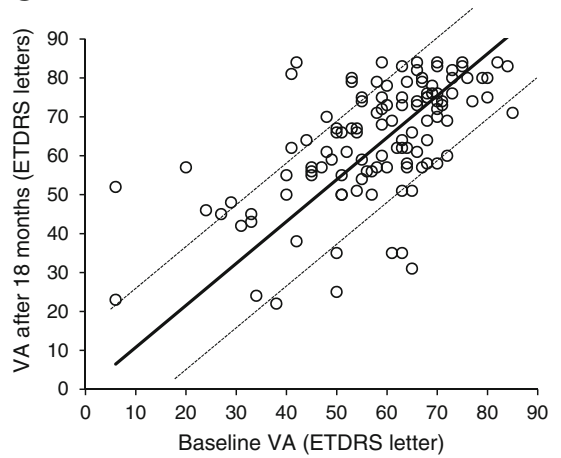

Month 18
B

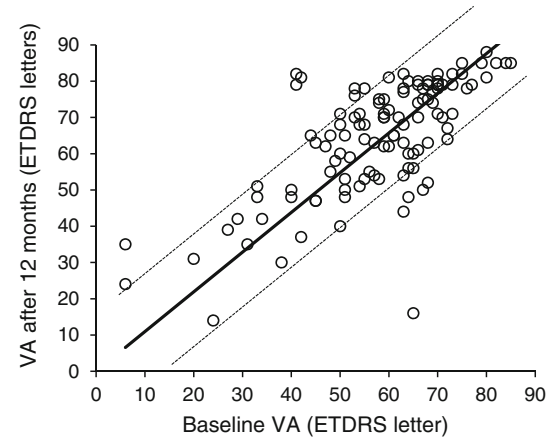

Month 12

$\mathrm{D}$

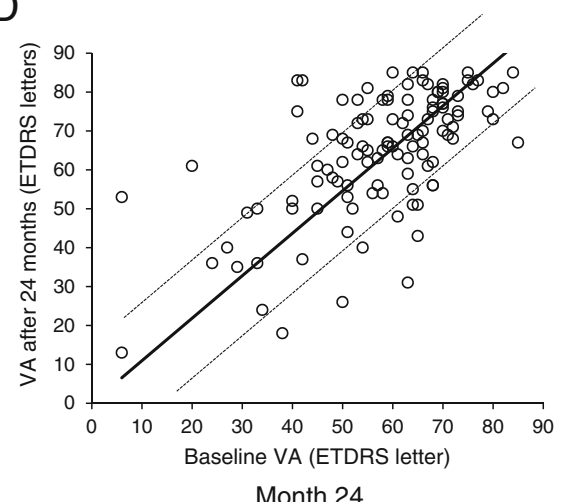

Fig. 2 Scatterplots of baseline VA vs VA at 4, 12, 18 and 24 months in eyes receiving treat-and-extend aflibercept for 2 years $(n=115)$ Points above the upper / below the lower dotted lines represent a gain / loss, respectively, of more than 15 ETDRS letters. ETDRS: Early Treatment Diabetic Retinopathy Study; VA: visual acuity 
Table 2 Mean VA and change in mean VA from baseline at 4, 12,18 and 24 months

\begin{tabular}{|c|c|c|}
\hline & Group A & Group B \\
\hline Number of patients & 105 & 33 \\
\hline \multirow[t]{2}{*}{ Number of eyes } & 115 & 33 \\
\hline & Baseline & \\
\hline VA (mean $\pm S D)$ & $57.9 \pm 14.9$ & $62.4 \pm 12.4$ \\
\hline \multirow[t]{2}{*}{$p$-value } & 0.0850 & \\
\hline & 4 months & \\
\hline VA (mean $\pm S D)$ & $63.6 \pm 14.5$ & $66.8 \pm 12.4$ \\
\hline Change in mean VA $(95 \% \mathrm{Cl})$ & 5.5 & 5.4 \\
\hline $95 \% \mathrm{Cl}$ & {$[3.7 ; 7.2]$} & {$[2.0 ; 8.7]$} \\
\hline$p$-value & $<0.0001$ & 0.0017 \\
\hline Difference in mean VA changes & 0.1 & \\
\hline $95 \% \mathrm{Cl}$ & {$[-3.7 ; 3.9]$} & \\
\hline \multirow[t]{2}{*}{$p$-value } & 0.9619 & \\
\hline & 12 months & \\
\hline VA (mean $\pm S D)$ & $64.6 \pm 15.8$ & $68.5 \pm 14.4$ \\
\hline Change in mean VA $(95 \% \mathrm{Cl})$ & 6.5 & 6.9 \\
\hline $95 \% \mathrm{Cl}$ & {$[4.4 ; 8.6]$} & {$[3.0 ; 10.9]$} \\
\hline$p$-value & $<0.0001$ & 0.0008 \\
\hline Difference in mean VA changes & -0.5 & \\
\hline $95 \% \mathrm{Cl}$ & {$[-5.0 ; 4.0]$} & \\
\hline \multirow[t]{2}{*}{$p$-value } & 0.8378 & \\
\hline & 18 months & \\
\hline VA $($ mean $\pm S D)$ & $64.0 \pm 15.0$ & $65.8 \pm 15.5$ \\
\hline Change in mean VA $(95 \% \mathrm{Cl})$ & 6.0 & 4.3 \\
\hline $95 \% \mathrm{Cl}$ & {$[3.7 ; 8.3]$} & {$[0.1 ; 8.6]$} \\
\hline$p$-value & $<0.0001$ & 0.0467 \\
\hline Difference in mean VA changes & 1.7 & \\
\hline $95 \% \mathrm{Cl}$ & {$[-3.1 ; 6.5]$} & \\
\hline \multirow[t]{2}{*}{$p$-value } & 0.4952 & \\
\hline & 24 months & \\
\hline VA (mean $\pm S D)$ & $64.8 \pm 15.6$ & $63.2 \pm 17.3$ \\
\hline Change in mean VA $(95 \% \mathrm{Cl})$ & 7.0 & 1.2 \\
\hline $95 \% \mathrm{Cl}$ & {$[4.7 ; 9.3]$} & {$[-3.1 ; 5.6]$} \\
\hline$p$-value & $<0.0001$ & 0.5733 \\
\hline Difference in mean VA changes & 5.7 & \\
\hline $95 \% \mathrm{Cl}$ & {$[0.8 ; 10.6]$} & \\
\hline$p$-value & 0.0224 & \\
\hline
\end{tabular}

Group A: eyes receiving the TE regimen for 2 years; Group B: eyes receiving the TE regimen in Year 1 and the PRN regimen in Year 2. VA values refer to least-squares means. Cl: confidence interval; PRN: pro re nata; SD: standard deviation; TE: treat-and-extend; VA: visual acuity

experiences with the proactive TE dosing regimens that allow extension of treatment intervals have fuelled the spread of the protocol among retinal specialists around the world $[12,20]$. The Preferences and Trends Survey indicates that almost $80 \%$ of US clinicians adhere to the TE approach, as do about $60 \%$ of their foreign counterparts [20].

In this retrospective observational study, intravitreal aflibercept therapy initiated under a TE regimen in treatment-naïve patients with nAMD was assessed over a period of 24 months. The mean baseline VA of $57.9 \pm$ 14.9 letters among patients who received TE therapy for 2 years in our study is slightly higher than that reported in other real-world studies [21-23]. This could introduce potential difficulty in demonstrating improvement in vision because, compared with eyes with lower VA at baseline, eyes with higher VA have reduced chance of gaining more but greater potential to lose vision [18]. We observed significant improvements in mean VA after the initial loading dose, which were maintained for the full 24 months of the study. There was a gain of 6.5 letters in mean VA after 1 year of treatment $(p<0.0001)$ and 7.0 letters after 2 years $(\mathrm{p}<0.0001)$. The proportion of eyes with VA of $\geq 70$ letters more than doubled after 12 months, and remained elevated after 24 months. In the natural course of nAMD, patients typically lose $\geq 3$ VA lines after 12 months [24]. Most eyes in our study had stable VA ( \pm 3 VA lines; dotted lines in Fig. 2) after treatment with intravitreal aflibercept under a TE regimen throughout the study period.

There are currently no data from RCTs on the outcomes of intravitreal aflibercept TE treatment for nAMD. In the VIEW studies, a fixed-dosing protocol was mandated during the first year and after this a "capped PRN" approach was used [25]. The 96-week results of the VIEW studies and our 2-year retrospective real-life study show comparable visual function outcomes, despite following different treatment approaches [25].

Intravitreal aflibercept TE therapy was investigated in the prospective Aflibercept Treat and extend for Less frequent Administration Study (ATLAS) which showed a median VA improvement of 7.5 ETDRS letters from baseline to 2 years in 31 treatment-naïve patients with nAMD, with mean numbers of injections of 8.0 and 6.5 during the first and second year, respectively [19]. That is similar to what we observed. Real-world data of intravitreal aflibercept TE therapy in a larger patient cohort have recently become available - the Fight Retinal Blindness Study Group has found a gain of 6.0 letters in mean VA from 136 eyes of 123 nAMD patients completing 24 months of follow-up in routine clinical practice [18]. The gain of 7.0 letters in mean VA in patients who received TE intravitreal aflibercept for 2 years in our study was slightly higher than the 6.0-letter gain observed by the Fight Retinal Blindness Study Group [18]. This may be in part attributed to the small difference in treatment frequency: the mean numbers of injections in the first 
Table 3 Subgroups of eyes according to VA at baseline, 4, 12 and 24 months

\begin{tabular}{lllllllll}
\hline VA, letters & \multicolumn{1}{l}{ Group A $(n=115)$} & \multicolumn{6}{l}{ Group B $(n=33)$} \\
\hline & Baseline & 4 months & 12 months & 24 months & Baseline & 4 months & 12 months & 24 months \\
& $\mathrm{n}(\%)$ & $\mathrm{n}(\%)$ & $\mathrm{n}(\%)$ & $\mathrm{n}(\%)$ & $\mathrm{n}(\%)$ & $\mathrm{n}(\%)$ & $\mathrm{n}(\%)$ & $\mathrm{n}(\%)$ \\
$\geq 70$ & $24(20.9)$ & $45(39.1)$ & $56(48.7)$ & $50(43.5)$ & $9(27.3)$ & $19(57.6)$ & $19(57.6)$ & $17(51.5)$ \\
$55-69$ & $51(44.3)$ & $47(40.9)$ & $29(25.2)$ & $40(34.8)$ & $17(51.5)$ & $7(21.2)$ & $8(24.2)$ & $5(15.2)$ \\
$35-54$ & $30(26.1)$ & $17(14.8)$ & $25(21.7)$ & $21(18.3)$ & $6(18.2)$ & $6(18.2)$ & $5(15.2)$ & $9(27.3)$ \\
$<35$ & $10(8.7)$ & $6(5.2)$ & $5(4.3)$ & $4(3.5)$ & $1(3.0)$ & $1(3.0)$ & $1(3.0)$ & $2(6.1)$ \\
\hline
\end{tabular}

$\mathrm{n}$ : number of eyes. Group A: eyes receiving the TE regimen for 2 years; Group B: eyes receiving the TE regimen in Year 1 and the PRN regimen in Year 2. PRN: pro re nata; TE: treat-and-extend; VA: visual acuity

and second year were 8.4 and 6.1 in our study, compared with 7.8 and 5.7 reported by the Fight Retinal Blindness Study Group [18].

Our study also provides real-world evidence demonstrating the long-term advantages of the TE approach over PRN in aflibercept-treated nAMD patients. There was no difference in mean VA gain at the end of the loading phase and at 12 months between treatment groups when the TE approach was used in all patients. This improvement in mean VA was maintained at 24 months in patients who continued the TE treatment throughout the 2 years, but lost in those who switched to PRN in the second year. Unsurprisingly, the difference in the mean number of injections per eye in Year 2 between the two treatment groups was apparent: 6.1 vs 2.5. It is interesting to note that the changes in the percentage of eyes with VA of $\geq 70$ letters from Year 1 to Year 2 were similar between the two groups. Among these eyes, one eye from Group A lost 18 letters as a result of a retinal pigment epithelial tear and one eye from Group B lost 40 letters due to the development of subfoveal atrophy; the rest of the eyes in both groups had VA changes of $<3$ VA lines. The changes in the proportion of eyes with VA of $\leq 54$ letters, however, followed different patterns in different treatment groups. Among those who followed the PRN protocol in the second year, the proportion of eyes with VA of $\leq 54$ letters increased from under one fifth at 12 months to one third at 24 months, suggesting that the decrease in mean VA from Year 1 to Year 2 in these eyes was mainly a result of an increase in the number of eyes with moderate to severe visual impairment. In comparison, in eyes that received the TE treatment throughout the 2 years, the proportion of those with VA of $\leq 54$ letters decreased slowly but steadily from baseline to 24 months, confirming the benefits of intravitreal aflibercept TE therapy in maintaining vision over time.

Our study is important because it provides real-world data from four centres in Slovenia where all intravitreal aflibercept treatments were conducted at the time, which fully represents a countrywide example of nAMD patient management. Based on our promising findings, intravitreal aflibercept is now being adopted in all seven anti-VEGF treatment centres in Slovenia. Additionally, the study results confirm the long-term benefits of intravitreal aflibercept using a TE regimen and add to the growing literature on the TE approach with anti-VEGF therapy which has been predominantly focused on the use of ranibizumab. The retrospective design of our study may also help to alleviate potential investigator bias which can sometimes be present in open-label studies. As with similar studies, the main limitations of our study are the observational and uncontrolled nature of the study design, as well as the inherent difference between baseline characteristics and disease progression in real-life patients compared with those in RCTs.

\section{Conclusion}

In this retrospective clinical study in real-life settings, intravitreal aflibercept treatment initiated under a TE regimen in treatment-naïve patients with nAMD led to significant visual improvement at 12 months, which was maintained at 24 months. TE therapy with intravitreal aflibercept proved to be a rational long-term strategy that can produce favourable outcomes in clinical practice.

\section{Abbreviations}

AE: Adverse event; ETDRS: Early Treatment Diabetic Retinopathy Study; nAMD: Neovascular age-related macular degeneration; PRN: Pro re nata; RCT: Randomised controlled trial; SD: Standard deviation; SD-OCT: Spectral domain optical coherence topography; TE: Treat-and-extend; VA: Visual acuity; VEGF: Vascular endothelial growth factor.

\section{Acknowledgements}

Medical writing and editorial assistance was provided by Dr. Zhizhi Fiske from Ketchum UK and supported by Bayer Consumer Care AG, Basel, Switzerland. The authors also thank Susanne Schwenke from Scossis GmbH for additional statistical support and all colleagues who were involved in treating the patients who were included in this retrospective study.

\section{Funding}

Bayer Consumer Care AG, Basel, Switzerland, provided funding for editorial support and additional statistical support. Bayer had no input into collecting and interpreting the data, or in writing the manuscript.

\section{Availability of data and material}

The datasets used and/or analysed during the current study are available from the corresponding author upon reasonable request. 


\section{Authors' contributions}

PJM, BG, CO and SP conceived the study, collected the data and performed the statistical analysis. PJM was a major contributor in writing the manuscript. All authors read the final draft, gave critical intellectual input and approved the final version.

\section{Ethics approval and consent to participate}

This retrospective, observational, multicentre study was conducted after requesting the approval of the National Medical Ethics Committee of Slovenia. We retrospectively identified in our medical records all patients who were started on intravitreal aflibercept between October 2013 and April 2015 for the indication of treatment-naiive $\mathrm{nAMD}$ in at least one eye. At the time all treatments were conducted in four centres, which provided data for this analysis. It is the participating hospitals' policy that, prior to starting treatment, all patients are requested to provide written consent to the use of their anonymised data for the purposes of clinical audit and research. All patients treated in Slovenia during this period, and included in this analysis, provided their written informed consent

\section{Consent for publication}

Not applicable for this section.

\section{Competing interests}

Prof Polona Jaki Mekjavic: honoraria for consultancy from Novartis, Bayer and Alcon. Dr. Bogdan Gregorčič and Dr. Slava Podgoršek: honoraria for consultancy from Novartis and Bayer. Dr. Cvetka Oberč does not have any conflict of interest with this submission.

\section{Publisher's Note}

Springer Nature remains neutral with regard to jurisdictional claims in published maps and institutional affiliations.

\section{Author details}

'Eye Hospital, University Medical Center Ljubljana, Grabloviceva 46, SI-1000 Ljubljana, Slovenia. ${ }^{2}$ Medical Faculty, University of Ljubljana, Ljubljana, Slovenia. ${ }^{3}$ Eye Department, General Hospital Dr. Franca Derganca, Nova Gorica, Slovenia. ${ }^{4}$ Eye Department, General Hospital, Novo Mesto, Slovenia.

${ }^{5}$ Eye Department, General Hospital, Celje, Slovenia.

Received: 22 May 2018 Accepted: 10 December 2018

Published online: 20 December 2018

\section{References}

1. World Health Organization: Causes of blindness and visual impairment. https://www.who.int/news-room/fact-sheets/detail/blindness-and-visualimpairment. Accessed 16 Dec 2018.

2. Brown MM, Brown GC, Stein JD, Roth Z, Campanella J, Beauchamp GR. Agerelated macular degeneration: economic burden and value-based medicine analysis. Can J Ophthalmol. 2005:40:277-87.

3. Schlottmann PG, Alezzandrini AA, Zas M, Rodriguez FJ, Luna JD, Wu L. New treatment modalities for Neovascular age-related macular degeneration. Asia Pac J Ophthalmol (Phila). 2017:6:514-9.

4. European Medicines Agency. 2017: Eylea Summary of Product Characteristics. http://www.ema.europa.eu/docs/en_GB/document_library/ EPAR_-_Product_Information/human/002392/WC500135815.pdf. Accessed 14 May 2018.

5. European Medicines Agency. 2011: Lucentis Summary of Product Characteristics. http://www.ema.europa.eu/docs/en_GB/document library/ EPAR_-_Product_Information/human/000715/WC500043546.pdf. Accessed 14 May 2018.

6. Brown DM, Michels M, Kaiser PK, Heier JS, Sy JP, lanchulev T, Group AS. Ranibizumab versus verteporfin photodynamic therapy for neovascular agerelated macular degeneration: two-year results of the ANCHOR study. Ophthalmology. 2009;116:57-65 e5.

7. Rosenfeld PJ, Brown DM, Heier JS, Boyer DS, Kaiser PK, Chung CY, Kim RY, Group MS. Ranibizumab for neovascular age-related macular degeneration. N Engl J Med. 2006:355:1419-31.

8. Heier JS, Brown DM, Chong V, Korobelnik JF, Kaiser PK, Nguyen QD, et al. Intravitreal aflibercept (VEGF trap-eye) in wet age-related macular degeneration. Ophthalmology. 2012;119:2537-48.
9. Ohr M, Kaiser PK. Aflibercept in wet age-related macular degeneration: a perspective review. Ther Adv Chronic Dis. 2012;3:153-61.

10. Zhang Y, Chioreso C, Schweizer ML, Abramoff MD. Effects of Aflibercept for Neovascular age-related macular degeneration: a systematic review and meta-analysis of observational comparative studies. Invest Ophthalmol Vis Sci. 2017:58:5616-27.

11. Spaide R. Ranibizumab according to need: a treatment for age-related macular degeneration. Am J Ophthalmol. 2007;143:679-80.

12. Gemenetzi M, Patel PJ. A systematic review of the treat and extend treatment regimen with anti-VEGF agents for Neovascular age-related macular degeneration. Ophthalmol Ther. 2017;6:79-92.

13. Hatz K, Prunte $C$. Treat and extend versus pro re Nata regimens of ranibizumab in neovascular age-related macular degeneration: a comparative 12 month study. Acta Ophthalmol. 2017;95:e67-72.

14. Johnston RL, Carius HJ, Skelly A, Ferreira A, Milnes F, Mitchell P. A retrospective study of Ranibizumab treatment regimens for Neovascular age-related macular degeneration (nAMD) in Australia and the United Kingdom. Adv Ther. 2017;34: 703-12.

15. Silva R, Berta A, Larsen M, Macfadden W, Feller C, Mones J, Group TS. Treatand-extend versus monthly regimen in Neovascular age-related macular degeneration: results with Ranibizumab from the TREND study. Ophthalmology. 2018;125:57-65.

16. Vardarinos A, Gupta N, Janjua R, Iron A, Empeslidis T, Tsaousis KT. 24-month clinical outcomes of a treat-and-extend regimen with ranibizumab for wet age-related macular degeneration in a real life setting. BMC Ophthalmol. 2017;17:58.

17. Wykoff CC, Ou WC, Croft DE, Payne JF, Brown DM, Clark WL, Abdelfattah NS, Sadda SR. Group T-AS: Neovascular age-related macular degeneration management in the third year: final results from the TREX-AMD randomised trial. Br J Ophthalmol. 2018;102:460-4.

18. Barthelmes D, Nguyen V, Daien V, Campain A, Walton R, Guymer R, et al. Two year outcomes of "treat and extend" intravitreal therapy using Aflibercept preferentially for Neovascular age-related macular degeneration. Retina. 2018;38:20-8

19. DeCroos FC, Reed D, Adam MK, Salz D, Gupta OP, Ho AC, Regillo CD. Treatand-extend therapy using Aflibercept for Neovascular age-related macular degeneration: a prospective clinical trial. Am J Ophthalmol. 2017:180:14250

20. Rezaei KA, Stone TW: 2014 Global Trends in Retina Survey: Chicago, IL. American Society of Retina Specialists. https://www.asrs.org/international/ global-trends-in-retina. Accessed 14 May 2018.

21. Eleftheriadou M, Vazquez-Alfageme C, Citu CM, Crosby-Nwaobi R, Sivaprasad S, Hykin P, Hamilton RD, Patel PJ. Long-term outcomes of Aflibercept treatment for Neovascular age-related macular degeneration in a clinical setting. Am J Ophthalmol. 2017;174:160-8.

22. Talks JS, Lotery AJ, Ghanchi F, Sivaprasad S, Johnston RL, Patel N, et al. Firstyear visual acuity outcomes of providing Aflibercept according to the VIEW study protocol for age-related macular degeneration. Ophthalmology. 2016; 123:337-43.

23. Warwick AN, Leaver HH, Lotery AJ, Goverdhan SV. Fixed bimonthly aflibercept in naive and switched neovascular age-related macular degeneration patients: one year outcomes. Int J Ophthalmol. 2016;9:1156-62.

24. Wong TY, Chakravarthy U, Klein R, Mitchell P, Zlateva G, Buggage R, Fahrbach K, Probst C, Sledge I. The natural history and prognosis of neovascular age-related macular degeneration: a systematic review of the literature and meta-analysis. Ophthalmology. 2008;115:116-26.

25. Schmidt-Erfurth U, Kaiser PK, Korobelnik JF, Brown DM, Chong V, Nguyen $\mathrm{QD}$, et al. Intravitreal aflibercept injection for neovascular age-related macular degeneration: ninety-six-week results of the VIEW studies. Ophthalmology. 2014;121:193-201. 